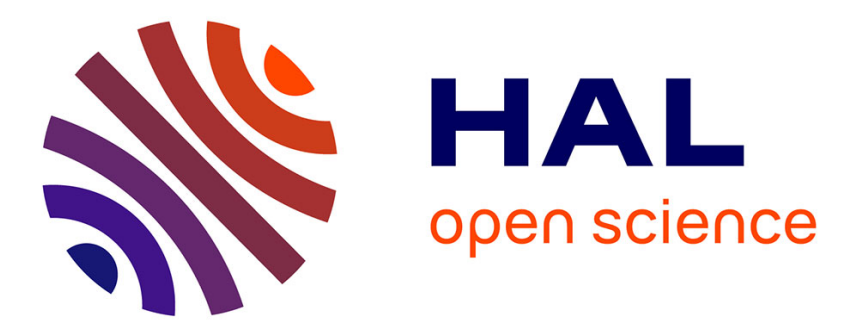

\title{
Study of the dielectric gradient values of reinforced concrete sample by considering radar measurements in an inverse problem
}

Xavier Ferrieres, Gilles Klysz, Vincent Guihard, Marius Albrand

\section{- To cite this version:}

Xavier Ferrieres, Gilles Klysz, Vincent Guihard, Marius Albrand. Study of the dielectric gradient values of reinforced concrete sample by considering radar measurements in an inverse problem. Inverse Problems in Science and Engineering, 2020, pp.1-15. 10.1080/17415977.2020.1763339 hal-02869515

\section{HAL Id: hal-02869515 https://hal.science/hal-02869515}

Submitted on 22 Jun 2020

HAL is a multi-disciplinary open access archive for the deposit and dissemination of scientific research documents, whether they are published or not. The documents may come from teaching and research institutions in France or abroad, or from public or private research centers.
L'archive ouverte pluridisciplinaire HAL, est destinée au dépôt et à la diffusion de documents scientifiques de niveau recherche, publiés ou non, émanant des établissements d'enseignement et de recherche français ou étrangers, des laboratoires publics ou privés. 


\title{
Study of the dielectric gradient values of reinforced concrete sample by considering radar measurements in an inverse problem.
}

\author{
X. Ferrieres ${ }^{1}$, G. Klysz ${ }^{2}$, V. Guihard ${ }^{3}$ and M. Albrand ${ }^{2}$ \\ ${ }^{1}$ ONERA/DEMR-LMA2S, Université de Toulouse, F-31055 Toulouse - France \\ ${ }^{2}$ Université de Toulouse, UPS, INSA, LMDC (Laboratoire des Matériaux et Durabilité des \\ Constructions), 135,Avenue de Rangueil, F-31 077 Toulouse Cedex 04, France \\ ${ }^{3}$ EDF R\&D- groupe Développement de Mesures Physiques, 78400 Chatou, France
}

\section{ARTICLE HISTORY}

Compiled February 28, 2020

\begin{abstract}
The goal of this study is to develop a tool that will evaluate the moisture content gradient in the depth of reinforced concretes in the context of building safety. The monitoring of ageing phenomena, such as the corrosion of steel reinforcements, depends on the amount of water and on its gradient as they are directly related to the dielectric values of the concrete. In this paper, to obtain these values, we propose to solve an inverse problem for reinforced concrete. First, an experimental device based on radar measurements is proposed with its 3D numerical model. Next, we describe an inverse process, to determine a dielectric profile according to the depth and validate it on measured data obtained for control samples.
\end{abstract}

\section{KEYWORDS}

Maxwell's equation, dielectric characterization of concrete, inverse problem, GPR

\section{Introduction}

Damage occurs frequently on civil engineering structures and diagnosis and prediction of its evolution require the knowledge of many parameters. One of the most important needs relates to the characterization of the properties of concrete gradients with depth. As concrete is a heterogeneous material both by its composition and by its exposure conditions, its properties vary with depth. It is essential to know the magnitude of the gradient of this variation, especially if the evolution of the material is to be predicted over decades. Most reliable predictive models also require knowledge, not only of the properties of a well-defined volume of the material, but also of how all these properties are distributed in depth. In particular, the monitoring of ageing phenomena : shrinkage, creep, internal swelling reactions (alkali-aggregate reaction and internal sulfate attack), reinforcement corrosion, etc., depend on the amount of water and on its gradients in the material [1]. Experiments in-situ show that this water content is directly related to the dielectric properties of the concrete [2], so evaluating the dielectric values of the reinforced concrete used in the inspected buildings is an important issue for civil engineering. At present, it is possible to determine the dielectric permittivity and conductivity of a homogeneous concrete by considering a $2 \mathrm{D}$ numerical model to solve an inverse problem from radar measurements [3]. The objective of this paper is to propose 
an inverse method to reconstruct dielectric profiles of a 3D reinforced concrete sample, according to depth. For this purpose, the aspects concerning the measurements, the modelling and the inversion are described. A first section presents the experimental device and the process used to perform the measurements. In a second section, we develop the numerical model that simulates the experimental process, with a strategy of calibration of the measurements in order to be able to compare them with computed values. Next, to validate this numerical model, a set of comparisons between simulations and measurements obtained by using several samples of concrete, is provided. In a third section with the measurement process and the numerical model proposed in the previous section, we describe the inverse problem implemented to determine dielectric profiles of the concrete from measurements. We give the mathematical formulation of the problem and the optimization algorithm to solve it. Next, by considering saturated concrete samples, with a numerical study of the cost function over the permissible range of the dielectric parameters, we show that there is a solution for our inverse problem. From measurements carried out on this type of concrete, we give several results of comparisons between values obtained by our inverse method and by measurements, which validate our process of inversion. Finally, we propose a dielectric model to take linear profiles of moisture inside reinforced concretes into account, for our inverse method and we compare the results with measurements.

\section{The experimental device}

Several systems based on radar technology exist to study the properties of the concretes used in buildings. For practical reasons, the LMDC has developed its own system [4] for taking measurements at several points on a structure in a relatively short time. This system consists of a device (a sensor) and a means of storage defined respectively by two 5100 GSSI antennas located inside a metallic box and an SIR30 GSSI computer to process the signal. Each GSSI antenna is composed of a transmitting and receiving dipole antenna having a center frequency of $1.5 \mathrm{GHz}$. The two GSSI antennas are completed with a survey wheel to determine the exact locations of the measurements. To illustrate the geometry of the device, Figure (1) gives a cross section where the different components can be seen.



Figure 1. Sensor device.

To use the data measured by this system in an inverse problem, it is necessary to simulate them and to have a numerical model of the system. This point is the subject of the next section. 


\section{Numerical model proposed to simulate the experimental system.}

\subsection{Numerical model}

The use of radar as a means of measurement for Non Destructive Testing in civil engineering is becoming increasingly widespread. Many papers proposing different models and numerical simulations of this means can be found in the literature, in particular for devices related to GSSI[5][3]. Each of these models describes the geometry of the device which uses dielectric and perfectly metallic materials, with for some of them, the possibility of having thin wires. In particular, our numerical model considers the two dipole-antennas in the GSSI antenna as 2 metallic planes connected by a thin wire, as indicated in Figure (2). A voltage generator and/or a linear circuit element, e.g. a resistor, are located on this wire to simulate the emission source. For the other components of the device like absorbers, we use different dielectric materials, which are also described in Figure (2). Next, to simulate the whole experimental system,
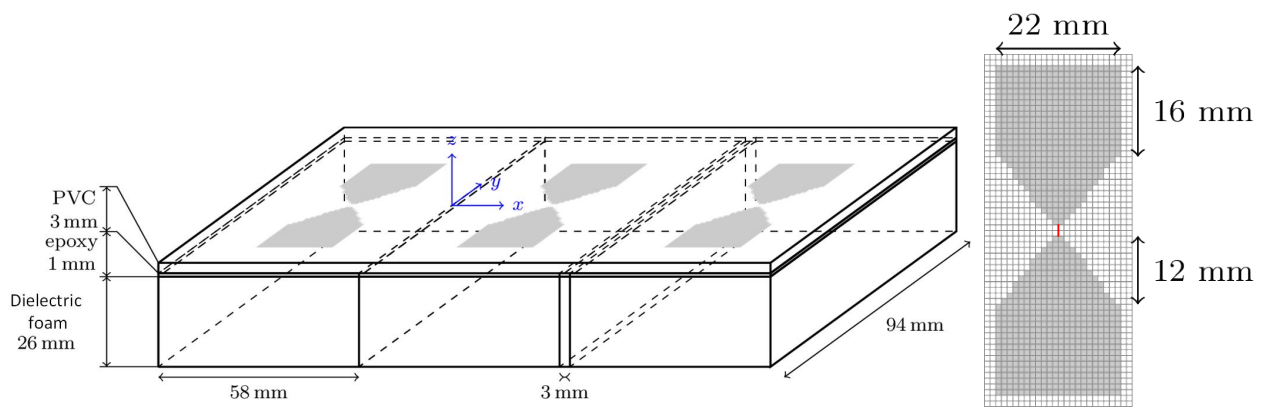

Figure 2. Description of the numerical model of the sensor device.

the device geometry described above is put in a computational domain $\Omega$ where the concrete sample is represented by a dielectric box. To simulate the experiment, the computational domain is bounded by a PML formalism [7] and some perfectly metallic conditions (see Figure 3). To obtain the resulting fields or voltages, we evaluate the

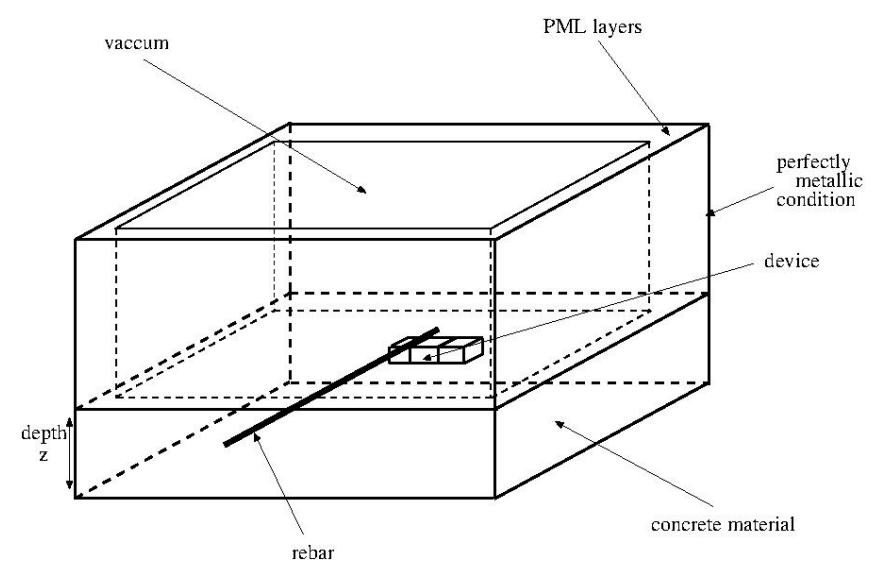

Figure 3. Experimental device.

currents $I$ and the electric fields $E$ by using the Maxwell equations coupled with a 
transmission line equation given by :

$$
\left\{\begin{array}{l}
\varepsilon \partial_{t} E+\sigma E+\mathbf{J}=\nabla \times H \\
\mu \partial_{t} H=-\nabla \times E \\
\partial_{t} I+R I=-\nu^{2} \partial_{l} q+\frac{V}{L d l}-\frac{\mathbf{E}}{L} \\
\partial_{t} q=-\partial_{l} I
\end{array}\right.
$$

where $E, H, I$ and $q$ are respectively the electric and magnetic fields in the computational domain $\Omega$ and the current and charge on the wire structures. The terms $\mathbf{E}$ and $\mathbf{J}=I / S$ are coupled terms which are given by the computed electric fields $E$ in $\Omega$ and the currents $I$ evaluated on the wires (see the Holland model [6]). $\nu$ defines the waves velocity in an environment characterized by its permittivity $\varepsilon$ and conductivity $\sigma$. The quantities $V$ and $R$ represent, respectively, a voltage generator and a resistor located on a wire segment. $S$ is a term homogeneous to a surface (see [6]). Finally, the quantities $L$ and $d l$ are, respectively, an inductance value given by the wire formalism [6] and the length of the segment where the voltage generator is located.

To complete the system of equations (1), a condition is added on perfectly metallic walls given by $n \times E=0$, where $n$ is the normal to the wall, and PML layers[7] are used to bound the computational domain $\Omega$. In this formalism, we set the condition $n \times E=0$ on the boundary $\partial \Omega$ of the domain $\Omega$. For the wire connected to the two metallic plates of the dipole-antennas, we set the condition $q=0$ at the two extremities of the wire.

For the initial conditions at $t=0$, all the fields, currents and voltages are assumed equal to 0 at every point of the computational domain. The equations (1) are solved by a finite difference method in the time domain [8], as in most papers dealing with the simulation of electromagnetic problems [9].

The major difficulty for the simulation of our measurement device is the lack of knowledge concerning the dielectric values of the absorbing components constituting the device, and also the source used for the emission. These are manufacturer's data that are not available. To define these unknowns, some authors have suggested solving an inverse problem from measured data[10]. Concerning our device, the parameters given by this approach for the same type of antenna are not optimal and a search by successive tests provided more satisfactory parameters. Finally, in our numerical model, the optimal choice for these parameters leads to :

- a resistor element of $R=1 e 5 \Omega$ on the wire of the emitting antenna ;

- a Gaussian pulse voltage generator $V(t)=e^{-\left(\frac{t-\mu}{\sigma}\right)^{2}}$ with $\mu=1 . e-9 s$ and $\sigma=0.13 e-9$. This provides a signal with a maximum of amplitude at the frequency $1.5 \mathrm{GHz}$ and a frequency range until $3 \mathrm{GHz}$ as it is observed in the measured data. In this choice, a Gaussian pulse also provides shapes of computed signals similar to the measured ones.

Despite these choices for the parameters, two major differences remain between the simulations and the measurements : the amplitude level and a time offset. To avoid this, we calibrate the measurements by making an additional one in free space. Then, from a simulation in free space, coefficients on the amplitude levels and time offset values between the measurement and the computation are determined for each receiver. These coefficients are then applied to the other measurements to match them with the simulation. 


\subsection{Comparisons between measurements and simulations}

Before using our numerical model of the measurement process, we validate it by comparing measurements and simulations for two configurations. For each configuration, we make two measurements on the receiver antennas $R_{1}$ and $R_{2}$ for a given location of the device in a open metallic box that is either empty or filled by a sample of reinforced saturated concrete (see Figure (4)). In the figure, $S_{1}$ defines the emitting antenna. In the experiments, the device is located at the centre of the box along the $y$ direction and the centre between $S_{1}$ and $R_{1}$ at $5 \mathrm{~cm}$ from the rebar along the $x$ direction.

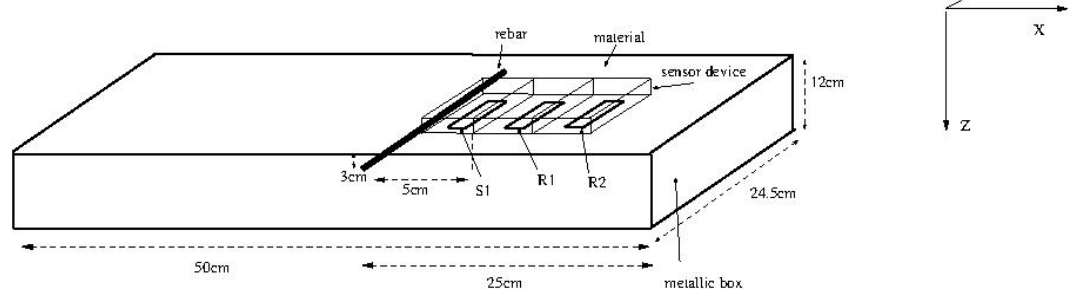

Figure 4. Experiment configuration.

In the first configuration, the metallic box is empty and, in the second, a reinforced concrete saturated with water is placed inside the box, its dielectric values having been experimentally determined previously by using an open-ended coaxial probe as described in subsection (3.3).

For the empty and filled configurations respectively, Figures (5) and (6) compare measurements with simulations for the signals obtained on both receivers, $R_{1}$ and $R_{2}$, of the device.

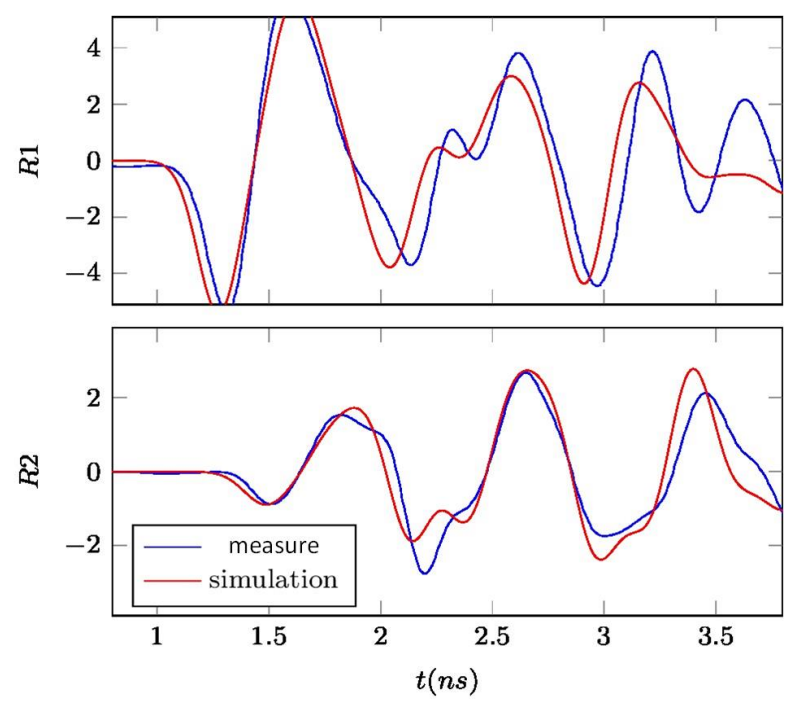

Figure 5. Comparison measurements/simulation (electric fields $(\mathrm{V} / \mathrm{m})$ ) for the box without material inside it.

For the first configuration, simulation and measurements give values that are not exactly the same but are very similar. This is particularly true for the first time steps. 

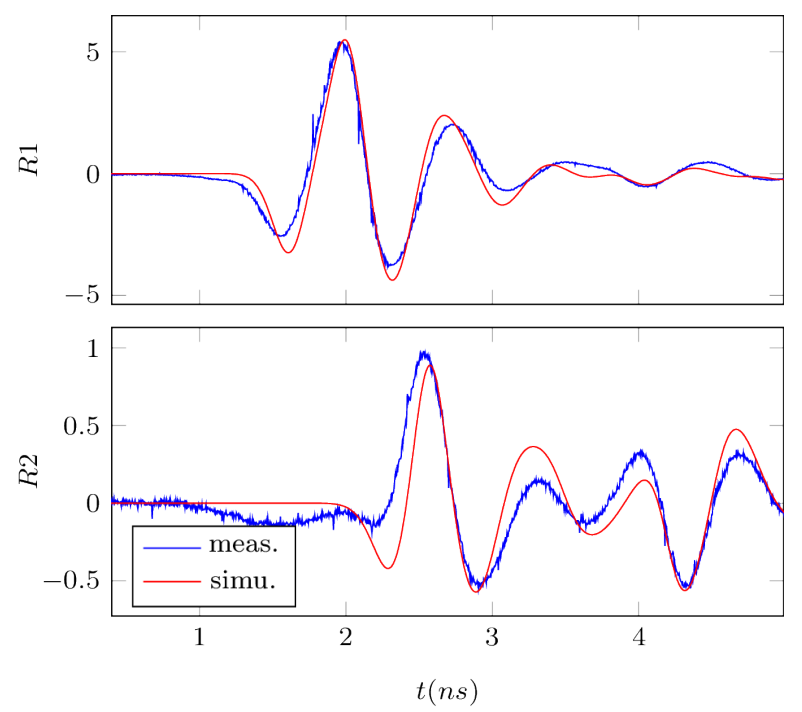

Figure 6. Comparison measurements/simulation (electric fields $(\mathrm{V} / \mathrm{m})$ ) with the box filled by saturated concrete.

Concerning the last configuration, if we take account of the fact that the dielectric parameters are not known exactly, the measurements and the simulated values can also can be considered very similar.

To conclude on all these comparisons, it can be said that our numerical model is not perfect but it represents reality sufficiently well to be used in an inverse process.

\subsection{Description of the open-ended coaxial probe}

An open-ended coaxial probe was used to directly compare our permittivity results. The principle of the measurement is based on the fact that dielectric electric constants of materials can be derived by analysing the transmission and reflection of electromagnetic waves through a sample inserted into a coaxial structure [17], or placed at the end of this structure in the case of devices based on reflection only. In this case, reflection phenomena are induced by an impedance discontinuity caused by the sample located at the end of the coaxial structure. Open-ended coaxial probes are one of many methods reviewed in [17] and [18]. They are simple to implement and have a broad frequency range driven by the network analyser used to synthesize the RF signal. The non-destructive and non-invasive characteristics of such tools have enabled different kinds of media to be investigated, such as flat surfaces of concrete [19]. In the present study, a specially designed large probe was built in order to investigate heterogeneous materials like concrete [20]. A picture of this probe with the vector network analyser (VNA) used to synthesize the electromagnetic signal is presented in Figure (7). The probe is characterized by a metal core of radius $a=6.5 \mathrm{~mm}$ and a dielectric insulator of radius $b=15 \mathrm{~mm}$ at the end of the probe. Such large diameters expand the investigated volume so that measurements can be performed on heterogeneous samples characterized by large representative elementary volumes. Also, because of the conical shape of the device, dimensions $a$ and $b$ vary along the probe length but the ratio $b / a$ remains constant, keeping the characteristic impedance of the probe constant and preventing any reflection within the probe. The device is then directly connected to a 


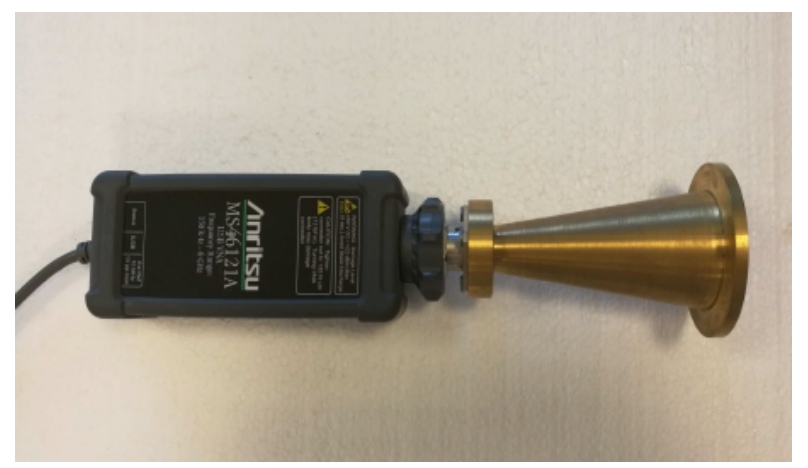

Figure 7. Open-ended coaxial probe.

vector network analyser (VNA). The analyser is used to synthesize and deliver the electromagnetic signal, which then propagates within the probe towards the material under test located at its end. The VNA also enables a reflection coefficient to be acquired, which is associated with the sample and can be related to its dielectric permittivity thanks to a capacitive model [18]. This model offers immediate permittivity estimation, but has a limited frequency range [21]. Using such a probe and capacitive model also requires a specific calibration procedure detailed in [22]. It consists in measuring the reflection coefficients of materials with known dielectric properties (air and Teflon here).

\section{Inverse process}

In this section, by using the numerical model defined in the previous section, we give the formulation of our inverse problem and the algorithm to solve it. Then, we apply the inverse process to measurements obtained on control samples saturated with water and with a linear variation of moisture according to the depth $z$ (see Figure 3).

\subsection{Formulation and optimization process to solve the inverse problem}

In this paper we are interested in evaluating the variation of permittivity and conductivity according to depth, for samples of reinforced concrete. The position of the bar in the concrete and its radius are assumed to be known. This hypothesis is quite admissible because the main function of a radar is to locate objects with high contrast in the observed environment. This is the case for rebars located inside the concrete. Thus, for our inverse problem, the unknowns are only the dielectric parameters $\varepsilon$ and $\sigma$ of the concrete. A linear profile of moisture according to the depth $z$ of a sample can be expressed as a linear profile of the speed of the waves $\nu(z)$ according to $z$ inside the sample [2]. Then, $\nu(z)$ can be written as :

$$
\nu(z)=(1-A(z)) \nu_{1}+A(z) \nu_{2}
$$


with $A(z)=\frac{z_{1}-z}{z_{1}-z_{2}}$ where $\left[z_{1}, z_{2}\right]$ defines the depth of the sample. The parameter $\nu(z)$ is related to the permittivity as :

$$
\varepsilon(z)=\frac{c_{0}^{2}}{\nu(z)^{2}}
$$

where $c_{0}$ defines the speed of the waves in the vacuum. Then, evaluating $\nu_{1}$ and $\nu_{2}$ is equivalent to determine $\varepsilon_{1}$ and $\varepsilon_{2}$ corresponding respectively to $\nu_{1}$ and $\nu_{2}$.

We choose to take the dielectric conductivity parameter $\sigma(z)$ as a constant value according to $z$. The measured fields are not very sensitive to this parameter and it would thus be difficult to obtain a conductivity value more accurate than an averaged value with our inverse process. The parameters of the problem are then given by the vector $x=\left(\varepsilon_{1}, \varepsilon_{2}, \sigma\right)$, and using these parameters the inverse problem can be written as :

$$
\min _{x}\left(\alpha_{1}\left\|E_{1}(x)-E_{m e s R 1}\right\|^{2}+\alpha_{2}\left\|E_{2}(x)-E_{m e s R 2}\right\|^{2}\right)
$$

where $E_{1}$ and $E_{2}$ are respectively the components of the electric field evaluated on the receivers $R_{1}$ and $R_{2}$, and $E_{m e s R 1}, E_{m e s R 2}$ the same for the measured data. The minimization problem is under the condition given by the system of the Maxwell equations coupled by a transmission line equation (1). The coefficients $\alpha_{1}$ and $\alpha_{2}$ are values taken between 0 and 1 , which allow us to weight the receivers $R_{1}$ and $R_{2}$ differently. In particular, the values obtained for the receiver $R_{2}$ are smaller than for the receiver $R_{1}$, due to the fact that $R_{2}$ is located at a greater distance from the transmitter than $R_{1}$. The weighting coefficients enable points $R_{1}$ and $R_{2}$ to make the same contribution to the cost function.

The literature offers a variety of ways to solve our inverse problem which are based on Gauss-Newton methods[12] and quasi-Newton methods[13]. Because the number of unknowns is small in our inverse problem, we chose a Levenberg-Marquardt method [14] to solve it. In this process, the gradients are evaluated using a Broyden approach [15] and an FDTD method is applied to solve the Maxwell equations. Globally, the process of optimization can be described by the following sequence [16] :

(1) it $=0 ; \gamma=2 ; \mathrm{x}=x_{0}$ where $x_{0}$ is an initial point

(2) $A=J(x)^{T} J(x)$ and $g=J(x)^{T} f(x) ; \lambda=\tau * \max _{i}\left(A_{i i}\right)$

(3) do while $\left(\left(\|g\|_{\infty} \leq \epsilon_{1}\right)\right.$ et $($ it $<$ itmax $\left.)\right)$

- $\mathrm{k}=\mathrm{k}+1 ; h=-(A+\lambda I)^{-1} g$

- if $\left(\|h\|>\epsilon_{2}\left(\|x\|+\epsilon_{2}\right)\right)$ then

$\circ x_{n}=x+h$

○ $\rho=\left(F(x)-F\left(x_{n}\right)\right) /(L(0)-L(h))$

$\circ$ if $\rho>0$ then

$-x=x_{n}$

- $A=J(x)^{T} J(x) ; g=J(x)^{T} f(x)$

- $\lambda=\lambda * \max \left(1 / 3,1-(2 \rho-1)^{3}\right) ; \gamma=2$

$\circ$ else

$-\lambda=\lambda * \gamma ; \gamma=2 * \gamma$ 
where

$x_{0}$ defines an initial point

$J=\nabla f(x)$ is the gradient of the function $f(x)$ which is equal to an electric field

$F(x)=\frac{1}{2}\left\|f(x)-f_{\text {mes }}\right\|^{2}$ is the cost function

$\left(\epsilon_{1}, \epsilon_{2}\right)$ define 2 given values to approximate the 0

$\tau=1 . e-3$ is a coefficient which permits to compute $\lambda$

In this process, we evaluate the gradients of the electric field with respect to the dielectric parameters at each step by a Broyden formula [15]. This evaluates the gradients at step $k$ of the optimization process by considering their previous values at step $k-1$ :

$$
J_{k+1}=J_{k}+\frac{\Delta F_{k} \cdot \Delta x_{k}^{T}-J_{k} \Delta x_{k} \cdot \Delta x_{k}^{T}}{\Delta x_{k}^{T} \cdot \Delta x_{k}}
$$

with $\Delta F_{k}=F\left(x_{k+1}\right)-F\left(x_{k}\right)$ and $\Delta x_{k}=x_{k+1}-x_{k}$.

It is known that, as the iterations increase, this process leads to an increasing error on the derivatives. To avoid this, after each $n$ iterations, the derivatives are evaluated numerically using a first order Taylor formula. The value of $n$ is chosen by the user. In our configurations, we chose to take $n=1$ and $n$ higher than the number of iterations necessary for the convergence of the optimization process. At convergence of the process, the result was the same, showing that, for our problem, this reinitialization of the derivatives was not very relevant to improve the convergence of the process. In addition, we did not take exactly the inverse but the pseudo-inverse of $A$ to avoid slow convergence in the vicinity of the solution due to an almost singular matrix $A$.

\subsection{Validation against measurements}

In order to validate our inverse process, two classes of rectangular prism samples were manufactured with two types of concrete.

If we define the term $W / C$ as the ratio of the weight of water to the weight of cement used in a concrete mix, the first class of samples is obtained by using a concrete with $W / C=0.5$ and the second class by using concrete with $W / C=0.7$. A higher ratio leads to higher porosity. So, in the case of saturated concrete, for the first class of samples noted $5 S N C$ a smaller amount of water or moisture is present than for the second class noted $7 S N C$. This implies higher dielectric values for $7 S N C$ than for $5 S N C$.

At the end of manufacturing, the samples are in a moisture saturated state and are considered as dielectrically homogeneous samples. Next, to modify their state and create a water gradient, we define a particular drying process that creates a linear moisture profile inside the sample, between its two surfaces and thus a linear profile of the waves speed values. To do this, all the faces of the samples are sealed except the two largest which are put in contact with air of controlled humidity until the weight of the samples stabilizes. In this state, the samples must be considered as heterogeneous dielectric materials.

By using these two kinds of samples, we produced ten configurations for making comparisons between measurements and the results obtained by the inverse process. The first three configurations, noted $7 S N C s 1,7 S N C s 2$ and $7 S N C s 3$, were obtained by exposing the measuring face of three similar $7 S N C$ samples to air with a relative 
humidity $(\mathrm{RH})$ of $65 \%$ and its opposite face to an $\mathrm{RH}$ of $100 \%$. To obtain the following three samples, we proceeded similarly by reversing the moisture levels between the two faces of the $7 S N C$ samples. We obtained the three configurations noted $7 S N C h 1$, $7 S N C h 2$ and $7 S C N C h 3$. The next two configurations noted $5 S C N s$ and $5 S C N h$ were obtained using two $5 S C N$ samples and exposing their measuring faces to $65 \%$ and $100 \% \mathrm{RH}$ and the opposite faces to $100 \%$ and $65 \% \mathrm{RH}$. Finally two configurations noted $7 S N C 81 s$ and $7 S N C 81 h$ were obtained by exposing two $7 S N C$ samples to $65 \%$ $\mathrm{RH}$, and $81 \%$ respectively on the measuring face and an $81 \% \mathrm{RH}$, respectively $65 \%$ on the opposite face.

Concerning the different samples generated, it is important to note that we do not know their dielectric values exactly, but we know several properties :

- the dielectric values for the saturated sample $7 S N C$ are larger than for the saturated sample $5 S N C$;

- the dielectric values for all the samples after drying are smaller than for the same samples in the saturated state.

To validate our inverse process, we must find these different properties for the samples processed.

We process all the proposed samples by first considering them in a saturated state, and then, as homogeneous dielectric materials. With this hypothesis, we begin by a numerical study to determine whether our inverse process, has a solution, that is to say if we have a global minimum for our cost function or not. To verify this point, different permittivity/conductivity pairs taken in the admissible domain of our physical material, are used to evaluate and draw this cost function for two types of saturated samples $7 S N C$ and $5 S N C$. Figure (8) shows these cost functions and we notice the existence of a global minimum on each curve. Consequently, our inverse problem has a solution and furthermore, these curves give some information for the choice of the initial point in the optimization process. In particular, the cost functions seem to be a Cartesian product of the variations of the cost functions according to the permittivity and the conductivity. In other words, both parameters seem to act independently on the cost functions. In fact, this is not exactly true and the minimum of each cost function is not equal to a permittivity and a conductivity pair, that are solutions of two 1D optimization problems. However, the shape of the cost functions can be used to define a good initial point. This has already been pointed out in the article [3] for another kind of measurement device. Figure (8) also shows that the variation according to the conductivity is smooth and convex while that according to the permittivity presents several local minima. Then, as proposed in [3], to obtain a good initial point, we take a value of conductivity $\sigma_{0}$ in its search interval and, by using a $1 \mathrm{D}$ global optimization process, evaluate a value of permittivity $\varepsilon_{0}$ that minimizes the cost function. The initial point for our optimization process is given by $\left(\varepsilon_{0}, \sigma_{0}\right)$. This strategy considerably reduces the number of iterations needed for the optimization process to converge.

Next, by using our inverse method on the saturated samples, with an initial point evaluated by the previous strategy, we obtain the results given in Table (1) and in Figure (9).

By considering the results given in the table and the figure, we can see that, for the permittivity parameter, the value is higher for concrete obtained with a $W / C=0.7$ than for those obtained with a $W / C=0.5$. This is in accordance with the expected results. However, this property is not so obvious for the conductivity, even though the values are rather smaller than the average of the others.

In addition, a permittivity measurement was made on the $7 S N C$ samples, giving 

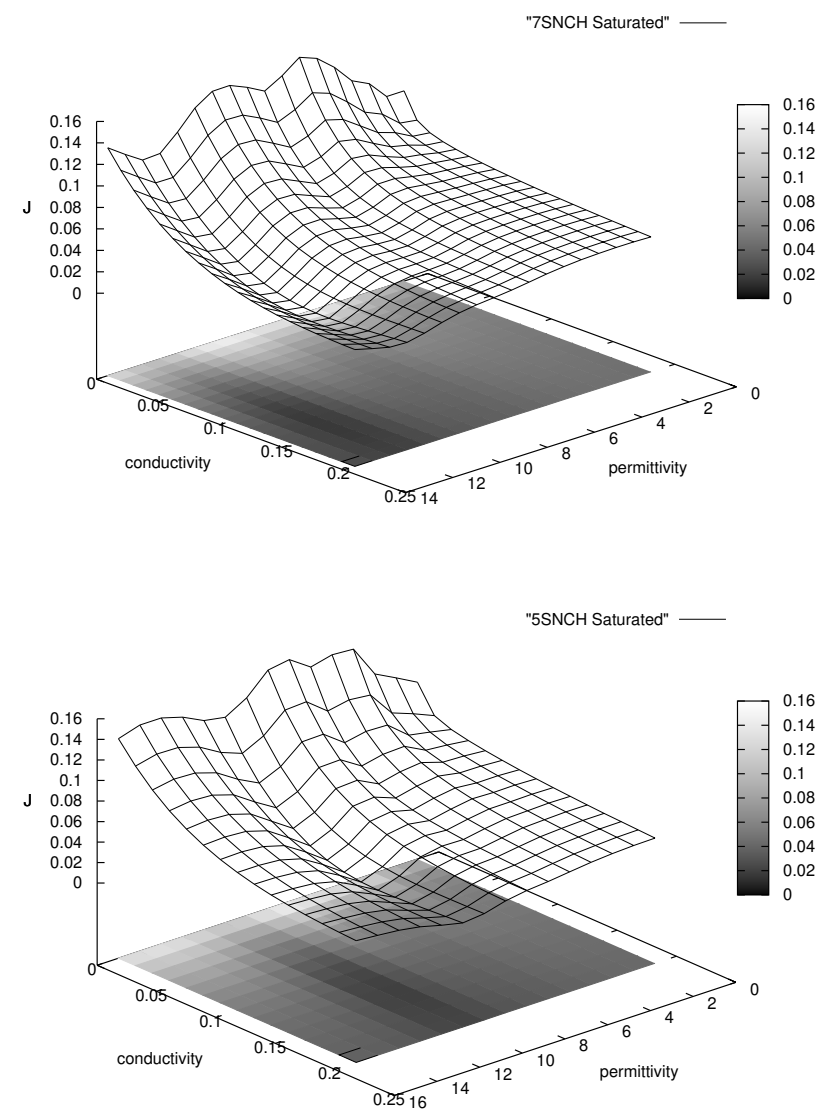

Figure 8. Variation of the cost function according to the permittivity and conductivity parameters for the samples $7 S N C$ and $5 S N C$ in a saturated state.
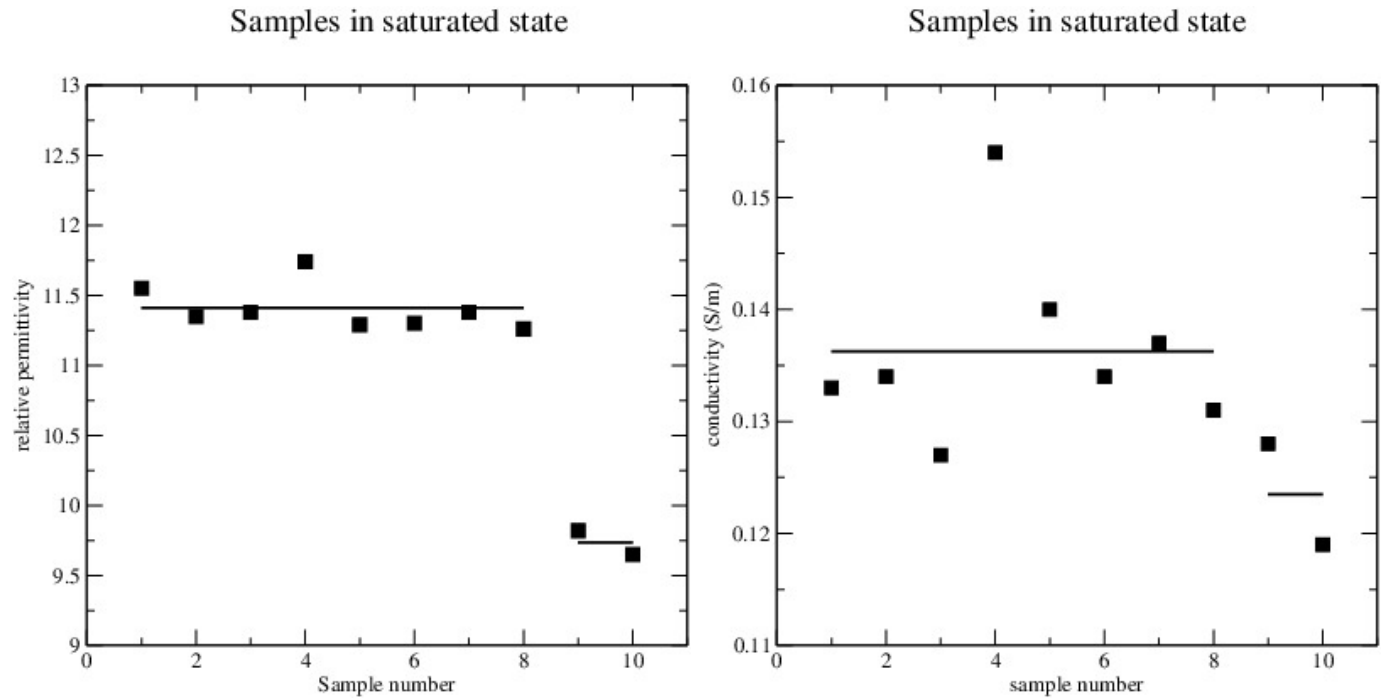

Figure 9. Dielectric permittivity and conductivity parameters found by inversion for samples in saturated state. 
Table 1. results obtained by inversion for all samples in saturated state.

\begin{tabular}{|c|c|c|c|}
\hline sample & sample number & $\varepsilon$ & $\sigma(S / m)$ \\
\hline 7SNCh1 & 1 & 11.55 & 0.133 \\
\hline 7SNCh2 & 2 & 11.35 & 0.134 \\
\hline 7SNCh3 & 3 & 11.38 & 0.127 \\
\hline 7SNCs1 & 4 & 11.74 & 0.154 \\
\hline 7SNCs2 & 5 & 11.29 & 0.14 \\
\hline 7SNCs3 & 6 & 11.3 & 0.134 \\
\hline 7SNC81h & 7 & 11.38 & 0.137 \\
\hline 7SNC81s & 8 & 11.26 & 0.131 \\
\hline 5SNCh & 9 & 9.82 & 0.128 \\
\hline 5SNCs & 10 & 9.65 & 0.119 \\
\hline
\end{tabular}

an averaged value of 11.33 which is near the value obtained by our inverse process (averaged value for solutions obtained is 11.41).

This measurement was performed with an open-ended coaxial probe.

All the results given by saturated samples show that our inverse process is very suitable for finding the dielectric parameters for an homogeneous saturated reinforced concrete.

Now, we are interested in finding the values of dielectric parameters of the sample after the drying process, by considering the concrete as a heterogeneous material. We recall that, in this paper, we consider only reinforced concrete samples with a linear variation of the moisture according to the depth. To evaluate the dielectric parameters, we use our inverse process by taking the initial point as the solution obtained for the same samples in the saturated state.

The results obtained by inversion and the measurements on the measuring surface by an open-ended coaxial probe (where the device is located) are given in Table (2). Figure (10) shows a comparison between the measured and computed values of the relative permittivity taken at the measuring surface. On these results, we notice that :

Table 2. Results obtained by inversion for all the samples after the drying process.

\begin{tabular}{|c|c|c|c|c|c|c|}
\hline sample & sample number & $\varepsilon_{1}$ & $\varepsilon_{2}$ & $\sigma(S)$ & $\varepsilon_{1}$ measured & relative gap for $\varepsilon_{1}$ \\
\hline 7SNCh1 & 1 & 11.25 & 7.24 & 0.109 & 10.33 & $8.91 \%$ \\
\hline 7SNCh2 & 2 & 10.29 & 9.21 & 0.117 & 10.67 & $-3.56 \%$ \\
\hline 7SNCh3 & 3 & 11.16 & 8.28 & 0.11 & 10.67 & $4.59 \%$ \\
\hline 7SNCs1 & 4 & 8.82 & 9.99 & 0.112 & 8.77 & $0.57 \%$ \\
\hline 7SNCs2 & 5 & 8.46 & 9.84 & 0.11 & 8.3 & $1.93 \%$ \\
\hline 7SNCs3 & 6 & 8.24 & 9.99 & 0.109 & 7.53 & $9.43 \%$ \\
\hline 7SNC81h & 7 & 8.29 & 7.16 & 0.113 & 8.07 & $2.73 \%$ \\
\hline 7SNC81s & 8 & 7.497 & 7.77 & 0.101 & 7.2 & $6.79 \%$ \\
\hline 5SNCh & 9 & 9.65 & 8.63 & 0.0714 & 9.9 & $-2.53 \%$ \\
\hline 5SNCs & 10 & 8.73 & 8.78 & 0.0737 & 8.6 & $1.51 \%$ \\
\hline
\end{tabular}

- the variation of the dielectric permittivity values are globally in good agreement with the variation of the moisture inside the samples studied;

- the results for the concrete samples in the saturated state and after drying are in accordance with the expected values. In particular, the dielectric and conductivity values for the saturated states are larger than after drying ;

- the values obtained by inversion and by measurements are in good agreement.

Considering all these points, it can be concluded that our inverse method seems to be a good approach for finding the moisture gradient inside reinforced concrete with a linear profile of moisture according to the depth. 


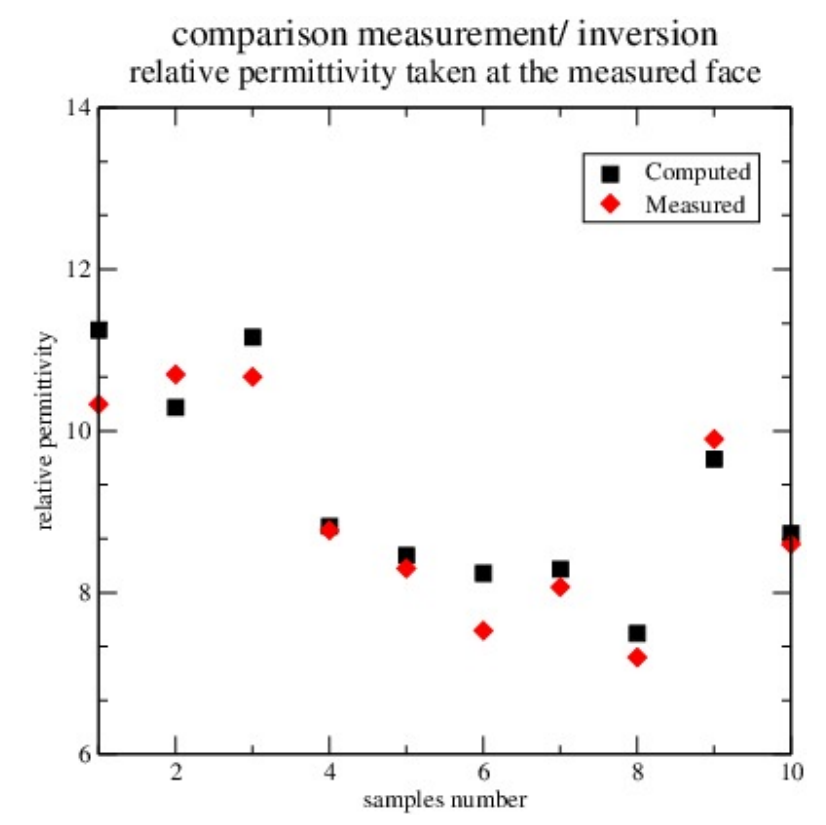

Figure 10. Comparison between measured and computed value for $\varepsilon_{1}$.

\section{Conclusion}

In this paper, we have presented a 3D inverse problem to evaluate dielectric parameters according to the depth, in reinforced concrete samples, by using radar measurements. First, we described the experiment device in detail and proposed a numerical model to simulate it. Next, by using this model, we defined an inverse problem to evaluate the moisture of a reinforced concrete as a linear function according to the depth. Several comparisons were made, with different samples of concrete, and demonstrated that, concerning the permittivity and the conductivity, the results obtained by inversion were in agreement with the dielectric characteristics of saturated samples. For samples with linear variation of the moisture according to depth, the results obtained for the permittivity present the same behaviour as the moisture, and then, it enables to go up to the gradients of water content in a sample of reinforced concrete. Moreover, the average relative gap between measured and computed dielectric permittivity is $3.04 \%$, which shows the ability of our inverse process to obtain the permittivity of the studied materials. Today, this point is an important advantage when buildings are to be monitored in a civil engineering context. In future investigations, the work presented here will also provide a good foundation for considering reinforced concrete by taking its carbonation into account with a nonlinear moisture profile according to the depth.

\section{Acknowledgements}

The authors gratefully acknowledge the French Agency of Research for their financial support of the CONTINUS project. Our thanks are extended to Susan Becker, a native English speaker, commissioned to proofread the final English version of this paper. 


\section{References}

[1] Alexander M., Bentur A., Mindess S. Durability of concrete, Design and Construction, CRC Press, 2017.

[2] Klysz G., Balayssac J.P. Determination of volumetric water content of concrete using ground-penetrating radar, Cement and Concrete Research, Vol.37, No.8, August 2007, pp.1164-1171.

[3] Ferrieres x., Klysz G., Mazet P., Balayssac J.P. Evaluation of the concrete electromagnetics properties by using radar measurements in a context of building sustainability, Computer Physics Communications 180 (2009) 1277-1281.

[4] Agred K., Klysz G., Balayssac J.P. Location of reinforcement and moisture assessment in reinforced concrete with a double receiver GPR antenna, Construction and Building Materials, Vol.188, Nov. 2018, pp.1119-1127.

[5] Giannopoulos A., Warren C. Creating finite difference time-domain models of commercial ground-penetrating radar antennas using Taguchi's optimization method, Geophysics, 76(2), 2011.

[6] Holland R. and Simpson L. Finite difference analysis of EMP coupling to thin struts and wires, IEEE Trans. on EMC, vol.23, pp.88-97, May 91.

[7] Berenger J.P. A Perfectly Matched Layer for the Absorption of Electromagnetic Waves, J. Comput. Phys. 114, 185-200, 1994.

[8] Yee K.S. Numerical solution of initial boundary value problems involving Maxwell's equation in isotropic media, IEEE Trans. Antennas Prop.,Vol.14,No.3,pp.302-307, May 1966.

[9] Taflove A. and Hagness S. Computational Electrodynamics : The Finite-Difference TimeDomain Method, Artech House, 2005.

[10] Warren C. Numerical modelling of high frequency ground-penetrating radars antennas, Thesis of the University of Edinburgh, 2009.

[11] Klysz G., Ferrieres X., Balayssac J.P., Laurens S. Simulation of direct wave propagation by numerical FDTD for a GPR coupled antenna, NDT\& E International 39 (2006), 338-347.

[12] Kelley C.T. iterative method for optimization, SIAM, 1999.

[13] Wright S.J., Nocedal J. Numerical optimization, Springer-Verlag, New-York inc, 1999.

[14] Gavin H.P. The Levenberg-Marquardt method for nonlinear least squares curves-fitting problems, Department of civil and environmental engineering, Duke University, 2017.

[15] Eyert V. A comparative study on methods for convergence acceleration of iterative vector sequences, J. Comput. Phys. 124, 271-285, 1996.

[16] Tingleff O., Madsen K., Nielsen H.B. Methods for non-linear least squares problems, 2nd edition, Informatics and mathematical modeling, Technical University of Denmark, 2004.

[17] Chen L.F., Ong C.K., Neo C.P., Varadan V.V and Varadan V.K. Microwave Electronics: Measurement and materials characterization, Wiley,2004.

[18] Stuchly M. and Stuchly S. Coaxial line reflection methods for measuring dielectric properties of biological substances at radio and microwave frequencies, IEEE transactions on Instrumentation and Measurement, Issue 29, 1980, pp. 176-183.

[19] Filali B., Boone F., Rhazi J. and Ballivy G. Design and calibration of a large openended coaxial probe for the measurement of the dielectric properties of concrete, IEEE Transactions on Microwave Theory and Techniques, issue 56, November 2008, pp. 23222328.

[20] Komarov S.A., Komarov A., Barber D.G., Lemes M. and Rysgaard S. Open-ended coaxial probe technique for dielectric spectroscopy of artificial grown see ice, IEEE Transactions on Geoscience and remote sensing, issue 54, august 2016, pp. 1-11.

[21] Guihard V., Taillade F., Balayssac J.P., Steck B. and Sanahuja J. Modelling the behaviour of an open-ended coaxial probe to assess the permittivity of heterogeneous dielectrics solids, PIERS 2017.

[22] Pozar D.M. Microwave Engineering, Vol 2, May 2004. 